Miranda-Mendoza, J.; Reynoso-Sánchez, L.F.; Hoyos-Flores, J.R.; Quezada-Chacón, J.T.; Naranjo, J.; Rangel-Colmenero, B. y Hernández-Cruz, G. (2020) Stress Score and LnrMSSD as Internal Load Parameters During Competition. Revista Internacional de Medicina y Ciencias de la Actividad Física y el Deporte vol. 20 (77) pp. 21-35 Http://cdeporte.rediris.es/revista/revista77/artstress1105.htm DOI: $10.15366 /$ rimcafd2020.77.002

\title{
ORIGINAL
}

\section{STRESS SCORE Y LnRRMSSD COMO PARÁMETROS DE CARGA INTERNA DURANTE UNA COMPETICIÓN}

\section{STRESS SCORE AND LnrMSSD AS INTERNAL LOAD PARAMETERS DURING COMPETITION}

\begin{abstract}
Miranda-Mendoza, J. ${ }^{1}$; Reynoso-Sánchez, L.F. ${ }^{\text {; }}$ Hoyos-Flores, J.R. ${ }^{3}$; QuezadaChacón, J.T. ${ }^{4}$; Naranjo, J. ${ }^{5}$; Rangel-Colmenero, B. ${ }^{6}$ y Hernández-Cruz, G. ${ }^{7}$

${ }^{1}$ Doctor en Ciencias de la Cultura Física. Profesora Facultad de Organización Deportiva, Universidad Autónoma de Nuevo León. Nuevo León (México) mmj5-7_12@hotmail.com

2 Doctor en Ciencias de la Cultura Física. Programa Educación Física y Ciencias del Deporte, Universidad Autónoma de Occidente, Sinaloa (México) felipe.reynoso@udo.mx

${ }^{3}$ Maestría en Actividad Física y Deporte con orientación en Alto Rendimiento. Profesor Facultad de Organización Deportiva, Universidad Autónoma de Nuevo León. Nuevo León (México) raul9991NBP@hotmail.com

${ }^{4}$ Doctor en Ciencias de la Cultura Física. Programa de Entrenamiento Deportivo, Instituto de Ciencias Biomédicas, Universidad Autónoma de Ciudad Juárez. Chihuahua (México) minaquezada21@gmail.com

${ }^{5}$ Doctor en Medicina. Departamento de Deporte e Informática. Universidad Pablo de Olavide. Sevilla (España) jonaore@gmail.com

${ }^{6}$ Doctora en Morfología. Facultad de Organización Deportiva, Universidad Autónoma de Nuevo León. Nuevo León (México) blanca.rangelc@uanl.mx

7 Doctor en Actividad Física y Calidad de Vida. Facultad de Organización Deportiva, Universidad Autónoma de Nuevo León. Nuevo León (México) german.hernandezcrz@uanl.edu.mx
\end{abstract}

\section{AGRADECIMIENTOS}

Este proyecto fue financiado por PRODEP DSA/103.15/15/6797.

Los autores agradecen al entrenador y los atletas por su apoyo para llevar a cabo la investigación.

Código UNESCO / UNESCO Code: 241106 Fisiología del Ejercicio / Exercise Physiology

Clasificación Consejo de Europa/Council of Europe Classification: 2. Bioquímica del Deporte / Biochemistry of Sport; 6. Fisiología del Ejercicio / Exercise Physiology; 17. Otras: Estrés fisiológico / Others: Physiological Stress.

Recibido 8 de marzo de 2018 Received March 8, 2018 Aceptado 21 de julio de 2018 Accepted June 21, 2018 


\section{RESUMEN}

El objetivo del estudio fue analizar el comportamiento del stress score (SS) y el logaritmo neperiano de la media de la raíz cuadrada de las diferencias de los intervalos sucesivos R-R (LnrMSSD) de la variabilidad de la frecuencia cardíaca (VFC) como indicadores de la carga interna mediante la modulación simpática y parasimpática, apoyado con parámetros bioquímicos de carga interna. Se evaluaron 14 atletas universitarios de balonmano (edad $22.30 \pm 1.83$ años). Se monitoreó la VFC y marcadores bioquímicos en seis momentos. Se analizaron las diferencias entre las tomas de cada variable mediante estadística descriptiva convencional y el tamaño del efecto con la $d$ de Cohen y la magnitud de Hopkins. Se examinaron las correlaciones de Pearson entre variables. EI LnrMSSD, SS y cortisol presentaron cambios significativos $(p<.05)$. Se encontraron correlaciones entre los parámetros de la VFC (SS y LnrMSSD) con creatin kinasa (CK) respectivamente. Los resultados del estudio muestran que el SS puede ser una metodología fiable para la evaluación de la carga interna durante una competición.

PALABRAS CLAVE: Estrés fisiológico, Sistema nervioso autónomo, Variabilidad de la frecuencia cardíaca, Marcadores biológicos, Carga interna, Balonmano.

\section{ABSTRACT}

The aim of this study was to analyse the behaviour of the stress score (SS) and the Neperian logarithmof the Root Mean Square of Successive R-R Interval differences(LnrMSSD) of heart rate variability (HRV) as indicators of internal load throughout sympathetic and parasympathetic modulation, supported by biochemical parameters of internal load. 14 handball university athletes (age 22.30 \pm 1 .83years) were evaluated. Six times of HRV and biochemical markers were collected. Each variable were analyzed by conventional statistics and using the Cohen's d, and Hopkins magnitude for the sample size effect. It was analyzed the Pearson correlations between variables. The LnrMSSD, SS and cortisol presented significant changes $(p<.05)$. Correlations were found between HRV (SS and LnrMSSD) and CK respectively. Results of this study shows that SS can be a reliable method for the evaluation of internal load during competition.

KEY WORDS: Physiological stress response, Autonomic nervous system, Heart rate variability, Biological markers, Handball, Internal load.

\section{INTRODUCCIÓN}

En el ámbito deportivo, el control de la carga interna es fundamental para maximizar las adaptaciones positivas al entrenamiento, las cuales ayudan en la realización de planificaciones adecuadas en competición y entrenamiento [1], además, proporciona información útil para determinar qué atletas están preparados para las 
exigencias de la competición [2]; por lo tanto, la cuantificación de la carga interna es considerado un tema relevante en el deporte [3].

Para la evaluación de la carga interna se han propuesto diferentes métodos, algunas investigaciones muestran relación entre $\mathrm{CK}$ y la urea con parámetros de intensidad y volumen del ejercicio [4]. La CK puede ser un determinante en la dosificación individual del entrenamiento en deportes intermitentes, en los cuales existen cargas elevadas y componentes de contracciones excéntricas [5]. Por otro lado, la urea indica el catabolismo de las proteínas lo que refleja que el volumen de la sesión de entrenamiento ha sido alto [5], también ha sido sugerido para el control del entrenamiento y evitar posibles adaptaciones negativas de las cargas físicas tanto en entrenamiento como en competición [4, 6]. Otro indicador de carga interna utilizado es el cortisol como uno de los principales glucocorticoides que prepara al organismo para responder a un estímulo interno o externo [7].Estos estímulos pueden ser ocasionados por estresores psicofisiológicos que se presentan por esfuerzos altos y continuos, los cuales han sido observados antes de un entrenamiento o de una competición como un indicador de respuesta anticipatoria $[8,9]$. La respuesta ante el estrés y las variaciones de cortisol pueden funcionar o no en sincronía dependiendo del grado de estrés psicofisiológico que se presente, y al mismo tiempo de la relación que tenga con el sistema nervioso autónomo [10, $11]$.

Estas evaluaciones bioquímicas (CK, urea y cortisol) han sido utilizadas en diversas investigaciones con éxito, sin embargo son de carácter invasivo, implicando cuidado especial en el manejo de las muestras sanguíneas y equipo especializado de alto costo [12].Por tal motivo, en la actualidad se están utilizando otros métodos no invasivos como la VFC, la cual se ha propuesto como una herramienta para la evaluación de la actividad del sistema nervioso autónomo (SNA) a través de la interacción entre el sistema simpático y parasimpático, manifestando la respuesta del corazón en diferentes demandas fisiológicas [13]. De esta forma la VFC proporciona información sobre la adaptación al entrenamiento [14], la fatiga y el estrés generado por las altas demandas físicas tanto en condiciones de entrenamiento como de la competición [15].

La VFC es un indicador sensible que mide las alteraciones provocadas por el estrés fisiológico y psicológico, ya que durante el ejercicio la actividad simpática se incrementa, mientras que en el período de recuperación se presenta una reactivación de la actividad parasimpática, este equilibrio refleja la restauración de la homeostasis cardiovascular importante en la recuperación global [16].En la práctica los índices más utilizados para monitorear las cargas de entrenamiento son la media de la raíz cuadrada de las diferencias de los intervalos sucesivos $R-R$ (rMSSD) y el diámetro transversal del diagrama de dispersión de Poincaré(SD1)que reflejan la actividad parasimpática [17], junto con el logaritmo neperiano de la rMSSD (LnrMSSD), que se está utilizando con gran frecuencia en valoraciones de equipo[1,18]. Por parte de la actividad simpática el parámetro que se acercan a una posible interpretación es el diámetro longitudinal del diagrama de dispersión de Poincaré (SD2) que es inverso a la actividad simpática. Para evitar este carácter 
inverso, Naranjo, et al. [13] propusieron en su lugar dos indicadores para la interpretación del diagrama de poincare denominados (SS) con el intención de obtener un valor directamente proporcional con la actividad simpática y el ratio simpático parasimpático (S:PS ratio), con el propósito de obtener una clara relación entre la actividad simpática y parasimpática que refleje el balance autonómico. Los autores mencionan que estos indices pueden servir como una herramienta para evaluar la asimilacion de las cargas de entrenamiento y competiciones en jugadores de futbol profesional[17].Dicho lo anterior, resaltamos que aunque existen estudios en diferentes deportes con el LnrMSSD, a la fecha no hay datos de aplicación del SS en otros deportes.

Por otra parte, en deportes de equipo donde las muestras suelen ser heterogeneas y de pequeño tamaño, se ha sugerido utilizar otro tipo de abordaje estadistico más útil que los valores de ppara la evaluación de los cambios significativos en un contrste de hipotesis [19], como el minimo cambio apreciable (SWC) de Hopkins, Marshall, Batterham, \& Hanin [20] y el tamaño del efecto (d) de Cohen.

El objetivo de nuestro estudio fue analizar el comportamiento del SS y el LnrMSSD como indicadores del estrés fisiologico simpático y parasimpático y su relación con marcadores bioquimicos durante una competición nacional en jugadores de balonmano y ver el aporte que brindanlos estadisticos de Cohen y Hopkins en el analisis de estas variables.

\section{MATERIAL Y MÉTODOS}

\section{SUJETOS}

14 atletas universitarios de balonmano(edad: $22.30 \pm 1.83$ años; estatura: $180.74 \pm$ $6.59 \mathrm{~cm}$; peso: 83,86 $\pm 14.80 \mathrm{~kg}$; porcentaje de grasa corporal: $18.51 \pm 8.22 \%$ ) pertenecientes al equipo representativo de la Universidad Autónoma de Nuevo León, todos con experiencia en competiciones nacionales participaron voluntariamente, fueron previamente informados del procedimiento a seguir y firmaron una carta de consentimiento contando con la aprobación del comité de bioética en Investigación en Ciencias de la Salud COBICIS (COBICIS801/2015/124-01HCG)

\section{PROCEDIMIENTO}

Previo al estudio todos los sujetos fueron sometidos a un examen completo rutinario que incluía un historial clínico, exploraciones y composición corporal mediante el método de absorciometría con rayos $X$ de doble energía (DXA) con la finalidad de descartar alguna enfermedad que afectara el propósito de la investigación.

Todas las evaluaciones se realizaron durante el campeonato universitario nacional, que es la competición más importante del equipo en su ciclo anual de entrenamiento. El LnrMSSD, SS, CK, urea y cortisol fueron evaluadosen las 
siguientes etapas: una semana antes a la competición (REP), la segunda se llevó a cabo un día previo al inicio de la competición (PRE), la tercerase tomó tras el último partido de la competición (FIN), la cual tuvo una duración de una semana acumulando seis partidos incluyendo la final a razón de un juego por día, la cuarta se realizó a la mañana siguientede finalizada la competición $(24 \mathrm{H})$, la quinta al segundo día $(48 \mathrm{H})$ y la sexta al tercer díade finalizada la competición $(72 \mathrm{H})$.

La cuantificación de CK, urea y cortisol se realizó mediante la recolección de muestras sanguíneas matutinas (8:00 a 9:00 horas con excepción de la toma Final la cual se realizó a las 16:00 horas por motivos del horario de la competición)las cuales se obtuvieron por veno punciónen tubos con anticoagulante EDTA (BD Vacutainer K2E/K2 de EDTA) de $4 \mathrm{~mL}$ siguiendo el procedimiento del CLSI (Clinical and LaboratoryStandardsInstitute, 2007), se centrifugaron a 3000 rpm durante 5 minutos para separar el plasma y se almacenaron a $-70^{\circ}$ hasta su procesamiento.

\section{ANÁLISIS DE LA CK Y UREA}

Se utilizaron tiras reactivas de creatin kinasa y de urea (Roche, Rotkreuz, Switzerland). Se utilizó el analizador Reflotron Plus (Reflotron Plus Roche,Rotkreuz, Switzerland).

\section{ANÁLISIS DE CORTISOL}

Se utilizó el método ELISA con el Kit Cortisol Human ELISA (abcam ab108665Cortisol Human ELISA Kit). Para el análisis se utilizó el protocolo establecido por el kit. Se preparó la curva de calibración y se colocaron junto con el control y las muestras de plasma en los pocillos específicos de la placa de ELISA. Los resultados del cortisol han sido previamente presentados en un estudio que analizó la relación entre el balance de estrés-recuperación psicológico y los niveles de cortisol [21].

\section{VARIABILIDAD DE LA FRECUENCIA CARDIACA}

Los registros de la VFC se realizaron en un ambiente controlado, utilizando el equipo Polar Team 2 (Polar Electro OY, Kempele, Finland), durante 15 minutos en posición supina, por la mañana, controlando el consumo de alimentos o medicamentos que pudieran alterar laVFC. Los datos obtenidos fueron examinados a través del software KUBIOS (University of Eastern Finland, Kuopio, Finland) utilizando el LnrMSSD (como variable del dominio de tiempo) y los ejes SD1 y SD2 del diagrama de dispersión de Poincaré.El cálculo del SS se realizó mediante la siguiente ecuación: $1000 \times 1 /$ SD2. Siguiendo el procedimiento propuesto por Naranjo et al.[13].

\section{ANÁLISIS ESTADÍSTICO}

Para el análisis estadístico de los datos se utilizó la versión 21 del paquete estadístico para ciencias sociales (SPSS Inc., Chicago, IL. USA). Los datos se presentan en media $(M)$ y desviación estándar $(D E)$, se realizó la prueba de 
normalidad utilizando el test de Shapiro-Wilk. Para realizar el análisis de comparación de medias se utilizó la prueba de ANOVA con el test de Tuckey como la prueba post-hoc y se realizó un análisis de correlación de Pearson para determinar las interrelaciones entre las variables. Para todos los análisis estadísticos se utilizó un nivel de significación $p<.05$. Se midió el tamaño del efecto (SE)mediante la d de Cohen, considerando los siguientes umbrales según Hopkinset al. [20]:0.1, cambio pequeño; 0.3moderado; 0.5 grande; 0.7 muy grande y 0.9 extremadamente grande.Siguiendo a Hopkins et al. [20] se consideró una d=0.2 como Mínimo Cambio Apreciable.

\section{RESULTADOS}

Tabla 1. Medias y DE de los resultados de las variables medidas en diferentes momentos.

\begin{tabular}{cccccc}
\hline & LnrMSSD & SS & CK & Urea & Cortisol \\
\hline REP & $4.22^{*} \pm 0.40$ & $7.51^{* \star} \pm 3.22$ & $202.88 \pm 119.32$ & $24.57 \pm 4.32$ & $189.48 \pm 72.90$ \\
PRE & $4.00 \pm 0.31$ & $10.74 \pm 3.17$ & $283.55 \pm 157.23$ & $29.22 \pm 9.06$ & $242.94 \pm 37.80$ \\
FIN & $3.62 \pm 0.61$ & $14.08 \pm 4.87$ & $788.50 \pm 706.68$ & $32.37 \pm 6.51$ & $162.22 \Delta \pm 69.83$ \\
$24 \mathrm{H}$ & $4.16^{\star} \pm 0.40$ & $9.46^{*} \pm 3.07$ & $641.31 \pm 560.50$ & $28.0 \pm 5.3$ & $150.09 \Delta \Delta \pm 53.05$ \\
$48 \mathrm{H}$ & $4.20^{*} \pm 0.50$ & $9.02^{*} \pm 4.01$ & $428.89 \pm 302.94$ & $30.48 \pm 9.87$ & $198.42 \pm 66.92$ \\
$72 \mathrm{H}$ & $4.30^{* *} \pm 0.49$ & $8.89^{\star} \pm 4.34$ & $284.57 \pm 114.94$ & $28.38 \pm 8.77$ & $175.18 \pm 64.10$ \\
\hline
\end{tabular}

Note: Se muestra medias $(M)$ y desviación estándar $(D E)$ de todas las variables analizadas. $C K=$ CreatinKinasa; $\mathrm{REP}=$ reposo; $\mathrm{PRE}$ = precompetición; FIN = final de la competición; $24 \mathrm{H}=24$ horas posteriores al final de la competición; $48 \mathrm{H}=48$ horas posteriores al final de la competición; $72 \mathrm{H}=72$ horas posteriores al final de la competición. ${ }^{*}=p<.05,{ }^{* *}=p<.01$ diferencias significativas con respecto a la toma FIN. $\Delta=p<.05 . \Delta \Delta=p<.01$ diferencias significativas con respecto a la toma PRE.

Tabla 2. Muestra el tamaño del efecto a través de la d de Cohen en las diferentes tomas de las variables analíticas.

\begin{tabular}{|c|c|c|c|c|c|c|}
\hline Variable & & REP & PRE & FIN & $24 \mathrm{H}$ & $48 \mathrm{H}$ \\
\hline \multirow[t]{5}{*}{ CK } & PRE & -0.58 & & & & \\
\hline & FIN & -1.42 & -1.17 & & & \\
\hline & $24 \mathrm{H}$ & -1.29 & -1.00 & 0.23 & & \\
\hline & $48 \mathrm{H}$ & -1.07 & -0.63 & 0.71 & 0.49 & \\
\hline & $72 \mathrm{H}$ & -0.70 & -0.01 & 1.23 & 1.06 & 0.69 \\
\hline \multirow[t]{5}{*}{ UREA } & PRE & -0.70 & & & & \\
\hline & FIN & -1.44 & -0.40 & & & \\
\hline & $24 \mathrm{H}$ & -0.71 & 0.17 & 0.74 & & \\
\hline & $48 \mathrm{H}$ & -0.83 & -0.13 & 0.23 & -0.33 & \\
\hline & $72 \mathrm{H}$ & -0.58 & 0.09 & 0.52 & -0.05 & 0.23 \\
\hline \multirow{5}{*}{ CORTISOL } & PRE & -0.97 & & & & \\
\hline & FIN & 0.38 & 1.50 & & & \\
\hline & $24 \mathrm{H}$ & 0.63 & 2.04 & 0.20 & & \\
\hline & $48 \mathrm{H}$ & -0.13 & 0.85 & -0.53 & -0.43 & \\
\hline & $72 \mathrm{H}$ & 0.21 & 1.33 & -0.19 & -0.43 & 0.35 \\
\hline
\end{tabular}

Nota: REP = Reposo; PRE = precompetición; FIN = final de la competición; $24 \mathrm{H}=24$ horas posteriores al final de la competición; $48 \mathrm{H}=48$ horas posteriores al final de la competición; $72 \mathrm{H}=$ 72 horas posteriores al final de la competición. 0.1 = Efecto pequeño; 0.3 = Efecto moderado; $0.5=$ Efecto largo; 0.7 = Efecto muy largo; 0.9 = Efecto extremadamente largo. 
En la Tabla 1 se muestranlos datos descriptivos obtenidos de las variables durante las diferentes tomas, incluyendo medias $(M)$, desviaciones estándar $(D E)$ y las respectivas significaciones de las variables analizadas, encontrando cambios principalmente en las variables LnrMSSD y SS, encontrando el mayor descenso y aumento respectivamente de sus valores en la toma FIN. En relación al cortisol se observaron diferencias en la toma $\mathrm{FIN}$ y $24 \mathrm{H}$, con respecto a la PRE, la cual presenta el pico más alto de este parámetro. En la Figura 1 se observa gráficamente el comportamiento de la LnrMSSD y SS a lo largo del registro.

La Tabla 2 muestra el tamaño del efecto de las variables analíticas (CK, urea y cortisol) en todas las tomas, en la CK se observan cambios en el tamaño del efecto en todas las toma, sin embargo los cambios más relevantes con tamaños de efecto extremadamente grandes $(<0.9)$ se presentaron en la toma $\mathrm{FIN}, 24 \mathrm{H}$ y $48 \mathrm{H}$ con respecto a la toma REP. Mientras que la urea encontró el cambio más relevante en la toma FIN con respecto a la toma REP con un tamaño del efecto de 1.44 considerado como extremadamente grande. $Y$ por último en lo que respecta a la variable del cortisol este mostro diferentes cambios en el tamaño del efecto con cambios extremadamente grandes en la toma PRE con respecto a la REP y en la toma FIN y $24 \mathrm{H}$ con respecto al PRE. Por otra parte en la Tabla 3 encontramos también el tamaño del efecto con respecto a los parámetros de VFC (LnrMSSD y SS). El LnrMSSD mostro cambios extremadamente grandes $(<0.9)$ en las toma FIN con respecto a la toma REP y a las $24 \mathrm{H}, 48 \mathrm{H}$ y $72 \mathrm{H}$ con respecto a la toma FIN. Mientras que el SS mostro sus mayores cambios en la toma PRE y FIN con respecto a la toma REP y $24 \mathrm{H}, 48 \mathrm{H}$ y $72 \mathrm{H}$ con respecto a la toma FIN.

Se encontraron correlaciones significativas de la CK con el SS $(r=0.265 ; p=.001)$ y el LnrMSSD $(r=0.329 ; p=.002)$. El resto de las variables no presentaron ninguna correlación significativa. 


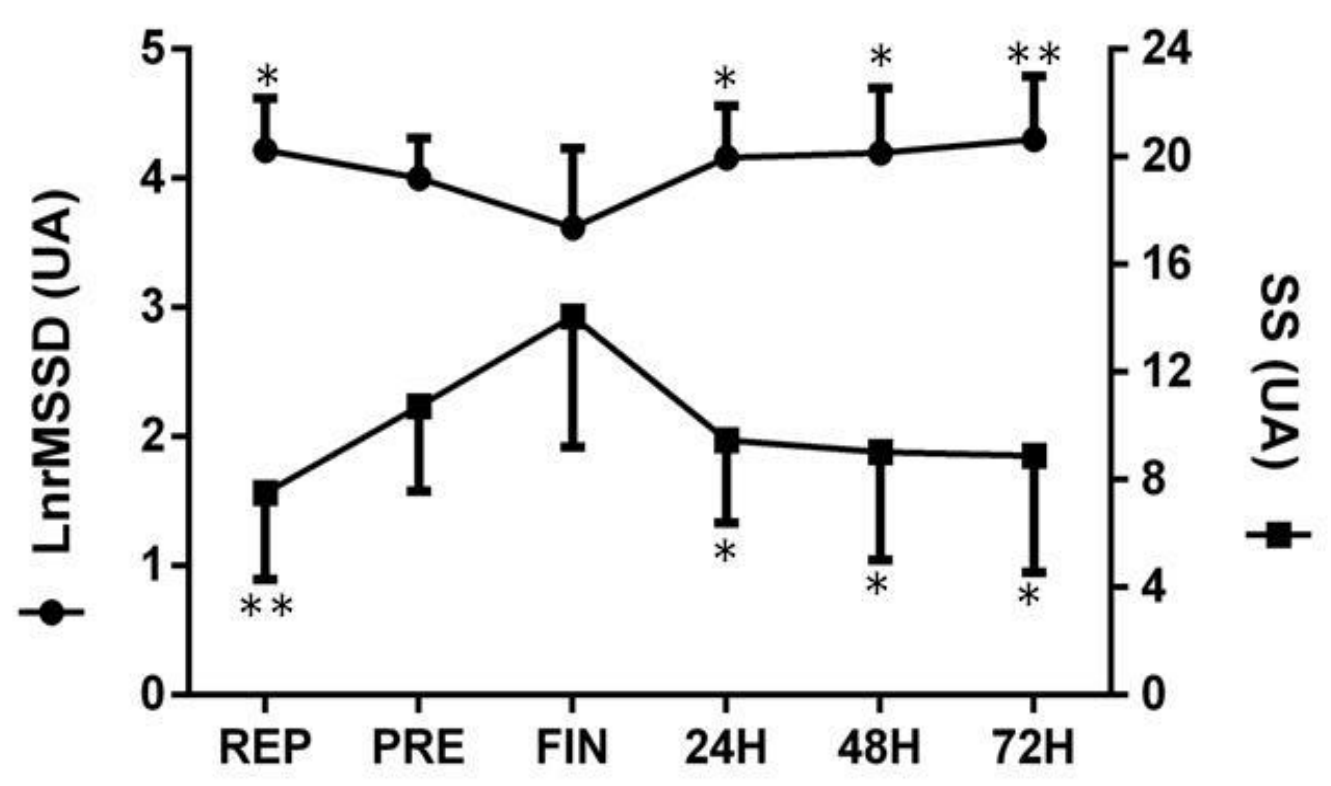

Figura 1. Se muestra la evolución de las variables LnrMSSD y SS durante las diferentes tomas. Nota: $\operatorname{Ln} r M S S D=$ Logaritmo neperiano de la raíz cuadrada del valor medio de la suma de las diferencias elevadas al cuadrado de los intervalos R-R; SS = índice de estrés. $\mathrm{REP}=$ reposo; $\mathrm{PRE}=$ precompetición; FIN = final de la competición; $24 \mathrm{H}=24$ horas posteriores al final de la competición; $48 \mathrm{H}$ $=48$ horas posteriores al final de la competición; $72 \mathrm{H}=72$ horas posteriores al final de la competición. Se marca con asteriscos las diferencias significativas observadas en cualquier variable con respecto a la medida de la toma FIN. ${ }^{*}=p<.05 ;{ }^{* *}=p<0,01$.

Tabla 3. Muestra el tamaño del efecto a través de la d de Cohen en las diferentes tomas de las variables de VFC (SS y LnrMSSD).

\begin{tabular}{ccccccc}
\hline Variable & & REP & PRE & FIN & $24 \mathrm{H}$ & $48 \mathrm{H}$ \\
\hline LnrMSSD & PRE & 0.62 & & & & \\
& FIN & 1.19 & 0.83 & & & \\
& $24 \mathrm{H}$ & 0.15 & -0.44 & -1.06 & -0.09 & -0.20 \\
\hline SS & 72H & 0.04 & -0.49 & -1.05 & -0.31 & \\
& PRE & 0.04 & -0.75 & -1.24 & & \\
& FIN & -1.01 & & & & \\
& $24 \mathrm{H}$ & -0.62 & -0.83 & & & \\
& $48 \mathrm{H}$ & -0.42 & 0.41 & 1.16 & 0.12 & 0.14 \\
\hline
\end{tabular}

Nota: $\mathrm{REP}=$ Reposo; PRE = precompetición; FIN = final de la competición; $24 \mathrm{H}=24$ horas posteriores al final de la competición; $48 \mathrm{H}=48$ horas posteriores al final de la competición; $72 \mathrm{H}=$ 72 horas posteriores al final de la competición. 0.1 = Efecto pequeño; 0.3 = Efecto moderado; $0.5=$ Efecto largo; 0.7 = Efecto muy largo; 0.9 = Efecto extremadamente largo.

\section{DISCUSIÓN}

La principal contribución del estudio fue a) presentar datos de SS y LnrMSSD en atletas de alto nivel universitario de balonmano durante una competición y b) mostrar una correlación significativa en los parámetros LnrMSSD y SS con la CK. 
Con respecto al SS encontramos en la toma REP valores que están dentro de los parámetros que Naranjo et al. [13] sugieren como normales (SS < 8). Al finalizar la competición se registró el mayor aumento de SS pudiendo considerarse como el período de mayor estrés fisiológico probablemente ocasionado por la acumulación de partidos, ya que conforme trascurrían los días de recuperación, los valores fueron descendiendo sin alcanzar el rango de normalidad propuesto por Naranjo et al. [13] para futbolistas. Aunque no hay valores de referencia en el balonmano nuestros datos mostraron un comportamiento similar al presentado por futbolistas los cuales tuvieron un seguimiento con estos parámetros durante una temporada completa, por lo cual coincidimos con Naranjo et al. [17] que el SS puede ser un indicador eficaz también en balonmano para el seguimiento de la carga de trabajo tanto en competición como en entrenamiento ya que el estrés generado por las cargas físicas a las que se someten los deportistas influyen directamente en el comportamiento de la actividad simpática.

Con respecto al LnrMSSD como indicador parasimpático se encontró una relación inversa con el SS como era de esperar puesto que reflejan actividades contrarias, notando también que los valores más bajos durante todas las mediciones fueron tomados al finalizar la competición reflejando el menor nivel de tono parasimpático, esto ocurre tras la acumulación de seis partidos en un corto periodo de tiempo incluyendo el partido de la semifinal y final, lo cual podría reflejar la acumulación de fatiga, no obstante en una medición única (como en este caso) no es posible diferenciar el estrés inducido por el partido previo de la suma de toda la carga de la competición.

En la literatura encontramos artículos centrados en la monitorización de los cambios sobre el rendimiento en deportes de equipo como el fútbol y balonmano mediante el LnrMSSD [22, 23].No encontramos estudios en situaciones posteriores a una competición para evaluar el comportamiento de la actividad parasimpática mediante esta variable, que resulta fiable para el seguimiento de atletas [24].Por lo contrario, sí hay estudios que reportan un descenso en la rMSSD al finalizar una competición de bádminton de máximo nivel por la acumulación de partidos [25], así como en sujetos tanto activos como sedentarios tras realizar una prueba de esfuerzo de $80 \%$ de intensidad [26]. Por otra parte consideramos importante analizar la recuperación en un periodo de descanso posterior a la competición, donde los resultados obtenidos sugieren que 72 horas es tiempo suficiente para regresar a condiciones basales, coincidiendo con Carvalho et al. [27], quienes proponen el mismo tiempo de recuperación para reducir el estrés fisiológico en jugadores de balonmano. Igualmente, Saboul et al.[3] recomiendan la medición pre y post competición de la rMSSD como un método de cuantificación de las cargas en condiciones reales de competencia. El LnrMSSD puede aportar información relevante y diferente sobre el estudio de la recuperación posterior a situaciones reales de competencia, sobre la de estudios que se enfocan sobre la respuesta del sistema nervioso autónomo en situaciones controladas [28]. 
El ejercicio intenso desencadena el aumento de marcadores biológicos, uno de los más utilizados en el deporte para la evaluación de la carga interna es la CK, que se libera al plasma cuando las fibras del músculo sufren un daño ocasionado por contracciones repetidas e intensas [29]. En nuestro estudio se encontró que los valores medios más elevados (Tabla 1 ) se registraron en la toma realizada al finalizar la competición en afinidad a lo reportado por Chatzinikolaou et al. [30] y de Moura et al. [31] en situaciones de competición o en entrenamientos, aunque estos valores no mostraron diferencia significativa entre ninguna de las tomas. Sin embargo, dado que la medida muestra alta variabilidad entre los sujetos con un Coeficiente de variación (CV) medio del $79.0 \%$, es poco útil la información basada en un contraste de hipótesis en una muestra tan pequeña y con tanta variabilidad. Este problema, muy frecuente en los deportes de equipo, fue analizado por Buchheit [19] sugiriendo el uso del tamaño del efecto de Cohen junto con el mínimo cambio apreciable propuesto por Hopkins et al.[20].

Aplicando estos estadísticos encontramos cambios relevantes en la $\mathrm{CK}$, con tamaños de efecto extremadamente grande, en las tomas $\mathrm{FIN}, 24 \mathrm{H}$ y $48 \mathrm{H}$ con respecto a la toma de reposo (Tabla 2), por lo cual deducimos que los cambios entre estas tomas independientemente de los valores de $p$, son relevantes y brindan información útil sobre el estado en que los atletas de balonmano se encontraban al finalizar la competición y en la recuperación.

Por otra parte, la CK mostró correlación con parámetros de VFC (SS y LnrMSSD), lo cual es un hallazgo importante debido a que son pocas las investigaciones que se han enfocado en analizar estas relaciones y en las que se han hecho no encontraron relación entre los parámetros de VFC y CK [6, 32]. Este hallazgo aunque no vienen a sustituir las mediciones de la CK por los de VFC, si muestra una mayor fuerza en la fiabilidad de los índices de VFC (LnrMSSD y SS) para el control de las cargas y la detección de fatiga.

En el estudio no se pudieron evaluar los distintos parámetros utilizados en la investigación (LnrMSSD, SS, CK urea y cortisol) durante toda la competición (partido a partido), por ello resulta difícil saber si la fatiga evaluada después del último partido era producto de una fatiga aguda que reflejaba el estímulo del partido final o la fatiga crónica por la acumulación de partidos. Considerado esto, se tomó en cuenta evaluar la urea, la cual se encuentra más ligada al volumen que a la intensidad [33], y aumenta sus valores en sangre cuando el ejercicio intenso se prolonga en el tiempo[34].

Los resultados dela urea en este estudio mostraron un comportamiento estable sin mostrar diferencias significativas en ninguna de las tomas. Sin embargo, al igual que ocurrió con la CK, el SE mostro cambios extremadamente grandes (d=1.44) en la toma FIN con respecto a la REP. Por tanto si aceptamos que existe un aumento relevante de la urea tras la final, podernos suponer que en el estrés y/o fatiga registrada tras la final tiene influencia la acumulación previa de partidos. 
La competición deportiva, implica una carga física, lo cual se ve reflejado en el incremento de estrés en los deportistas. Dicho estrés afecta al organismo a nivel hormonal, provocando un desequilibrio en el eje hipotalámico-pituitario-adrenal (HPA) y el sistema nervioso autónomo lo que puede reflejarse en un incremento en los niveles de cortisol y la respuesta cardíaca $[10,11]$. En el presente estudio, los valores basales de cortisol se encuentran dentro de los valores normales de reposo coincidiendo con lo reportado en otros estudios $[8,35]$.

Entre las tomas REP y PRE no hay cambio significativo, pero nuestros resultados muestran un tamaño de efecto extremadamente grande $(d=.97)$ posiblemente relacionado con una respuesta anticipatoria para de adaptar al organismo a las exigencias que demandará dicha competición, como se ha reportado en tenistas, jugadores de voleibol, baloncesto y golfistas [7, 9, 36]. Este comportamiento mostrado por los jugadores de balonmano se puede ver asociado al grado de dificultad de la competición, tal y como ocurre en jugadores de rugby [37].

Los niveles de cortisol disminuyen en la toma FIN de la competición significativamente $(p<.05)$ y con tamaño de efecto extremadamente grande $(\mathrm{d}$ $=1.50$ ) con respecto a PRE, así mismo una disminución con un tamaño de efecto pequeño $(d=0.38)$ con respecto a REP. En resumen, la toma REP se encuentra valores basales, posteriormente un aumento en la toma PRE $(\mathrm{d}=.97)$ y después un descenso en la toma FIN con respecto a REP $(d=0.38)$ y PRE $(p<.05 ; d=1.50)$ pudiendo reflejar una variación del patrón del ciclo circadiano de la secreción del cortisol, que manifiesta sus valores máximos en la mañana y disminuyendo constantemente durante el día [10], puesto que toma FIN tiene lugar a una hora diferente al resto (12:00pm).

Sin tener en cuenta la hora de la toma, no sería lógico un descenso del cortisol tras el partido, ya que los jugadores deberían encontrarse bajo el estrés desencadenado por la competición. Es necesario por tanto, corregir de alguna forma este efecto. $\mathrm{Si}$ consideramos que los valores de normalidad del cortisol plasmático a las 8:00am llegan hasta $250 \mathrm{ng} / \mathrm{dL}$ y a las $12: 00 \mathrm{pm}$ hasta $150 \mathrm{ng} / \mathrm{dL}$, podemos observar (Tabla 1) que el valor medio de reposo (REP) se encuentra al $80 \%$ del límite de normalidad, el PRE al $97 \%$ y el valor FIN se encuentra al $108 \%$ de su límite de normalidad. Esto indicaría que con independencia de la hora, el cortisol en la toma FIN puede considerarse cualitativamente más elevado que el PRE. Esto sería coherente con el análisis de Weitzman y colaboradores en 1971 (como se citó en Chan \& Debono [38] y Maidana, Bruno \& Mesch [39]), con la idea de que no son comparables cifras tomadas a diferentes horas [40].

Por otra parte, las respuestas fisiológicas a través de los niveles de cortisol y los parámetros LnrMSSD y SS no presentaron correlación estadísticamente significativa entre ellos, aunque el comportamiento del SS y de cortisol es similar entre las tomas REP y PRE, pudiéndose deber dicho comportamiento en ambas variables a una respuesta adaptativa del estrés fisiológico [40]. 
De acuerdo con McLean, Coutts, Kelly, McGuigan, y Cormack [41] acentuamos la importancia que tiene el balance entre la fatiga y la recuperación de los atletas para disminuir el riesgo de un descenso en el rendimiento deportivo, especialmente en periodos de competición. En nuestro estudio posterior a una competición los marcadores de estrés fisiológico se ven altamente comprometidos, por lo que es importante la evaluación de marcadores de fatiga para el monitoreo de la carga interna.

El presente estudio no estuvo exento de limitantes, dentro de las cuales las principales fueron: (1) La toma FIN realizada al finalizar la competición en un horario diferente al de las demás tomas, sin embargo medir el estado en el que terminaban los atletas era una de las prioridades del estudio, por lo cual asumimos esta limitación.(2) En este estudio no fue posible realizar un seguimiento de los diferentes parámetros en cada uno de los partidos, debido a las características de la competencia, donde los atletas disponen de poco tiempo para el análisis.

\section{CONCLUSIONES}

El comportamiento del SS pudo utilizarse como un buen indicador para la evaluación del estrés fisiológico simpático, al igual que el LnrMSSD nos proporcionó información sobre la recuperación mediante la actividad del tono parasimpático tras una competición nacional en los jugadores de balonmano de este estudio. Los parámetros de VFC no mostraron correlación con los marcadores biológicos a excepción de la CK. El análisis estadístico convencional por hipótesis nula no da información relevante sobre los cambios significativos de una competición a diferencia de los estadísticos de Cohen y Hopkins que aportan información sobre los cambios relevantes.

\section{REFERENCIAS BIBLIOGRÁFICAS}

1. Buchheit M, Racinais S, Bilsborough JC, Bourdon PC, Voss SC, Hocking J, Cordy $J$, Mendez-Villanueva A, Coutts AJ. Monitoring fitness, fatigue and running performance during a pre-season training camp in elite football players. J Sci Med Sport. 2013;16(6): 550-555. https://doi.org/10.1016/j.jsams.2012.12.003

2. Halson SL. Monitoring training load to understand fatigue in athletes. Sports Med.2014;44(supp 2): 139-147. https://doi.org/10.1007/s40279-014-0253-z

3. Saboul D, Balducci P, Millet G, Pialoux V, Hautier C. A pilot study on quantification of training load: The use of HRV in training practice. Eur $\mathrm{J}$ Sport Sci.2016;16(2): 172-181. https://doi.org/10.1080/17461391.2015.1004373

4. Díaz V, Díaz E, Peinado AB, Benito PJ, Calderón FJ, Sampedro J. Control biológico del sobreentrenamiento en un mesociclo precompetitivo en triatletas de élite: un estudio piloto. Archivos de Medicina Del Deporte. 2010;27(135), 31-40.

5. Calderon FJ, Benito PJ, Melendez A, González M. Control biológico del entrenamiento de resistencia. Revista Internacional de Ciencias del Deporte. 2006;2(2), 65-87. https://doi.org/10.5232/ricyde2006.00205 
6. Sarabia E, de la Cruz B, Naranjo J. Estudio comparativo de los perfiles semanales de creatin kinasa, urea y variabilidad de la frecuencia cardiaca en remeros de élite españoles. Archivos de Medicina Del Deporte. 2012;29(152), 952-958.

7. Edwards DA, Wetzel K, Wyner DR. Intercollegiate soccer: Saliva cortisol and testosterone are elevated during competition, and testosterone is related to status and social connectedness with teammates. Physiol Behav. 2006;87(1), 135-143. https://doi.org/10.1016/j.physbeh.2005.09.007

8. Coetzee N. Measurement of heart rate variability and salivary cortisol levels in beginner scuba divers. African Journal for Physical, Health Education, Recreation and Dance. 2011;17(4), 719-742.

9. Edwards DA, Kurlander LS. Woman's intercollegiate volleyball and tennis: Effects of warm-up, competition, and practice on saliva levels of cortisol and testosterone. Hormones and Behavior. 2010;58(4), 606-613. https://doi.org/10.1016/i.yhbeh.2010.06.015

10. Looser RR, Metzenthin P, Helfricht S, Kudielka BM, Loerbroks A, Thayer JF, Fischer JE. Cortisol is significantly correlate with cardiovascular responses during high levels of stress in critical care personnel. Psychosomatic Medicine. 2010;72(3), 281-289. https://doi.org/10.1097/PSY.0b013e3181d35065

11. Marques AH, Silverman MN, Sternberg EM. Evaluation of stress systems by applying noninvasive methodologies: Measurements of neuroimmune biomarers in the sweat, heart rate variability and salivary cortisol. Neuroimmunomodulation.2010;17(3), 205-208. https://doi.org/10.1159/000258725

12. Bocanegra OL, Diaz MM, Teixeira RR, Soares SS, Espindola FS. Determination of the lactate threshold by means of salivary biomarkers: Chromogranin A as novel marker of exercise intensity. Eur J Appl Physiol. 2012;112(9), 31953203. https://doi.org/10.1007/s00421-011-2294-4

13. Naranjo J, De La Cruz B, Sarabia E, De Hoyo M, Domínguez-Cobo S. Two new indexes for the assessment of autonomic balance in elite soccer players. Int J Sports Physiol Perform. 2015;10(4), 452-457. https://doi.org/10.1123/ijspp.2014-0235

14. Buchheit M, Mendez-Villanueva A, Quod MJ, Poulos N, Bourdon P. Determinants of the variability of heart rate measures during a competitive period in young soccer players. Eur J Appl Physiol. 2010;109(5), 869-878. https://doi.org/10.1007/s00421-010-1422-x

15. Wallace LK, Slattery KM, Coutts AJ. A comparison of methods for quantifying training load: Relationships between modelled and actual training responses. Eur J Appl Physiol. 2014;114(1), 11-20. https://doi.org/10.1007/s00421-0132745-1

16. Almeida AC, Machado AF, Albuquerque MC, Netto LM, Vanderlei FM, Vanderlei LC. Junior JN, Pastre CM. The effects of cold water immersion with different dosages (duration and temperature variations) on heart rate variability postexercise recovery: A randomized controlled trial. J Sci Med Sport. 2015;2440(15), 676-681. https://doi.org/10.1016/.j.jsams.2015.10.003 
17. Naranjo J, De la Cruz B, Sarabia E, De Hoyo M, Dominguez-Cobo S. Heart rate variability: a follow-up in elite soccer players throughout the season. Int J Sports Med.2015;36(11), 881-886. https://doi.org/10.1055/s-0035-1550047

18. Plews DJ, Laursen PB, Kilding AE, Buchheit M. Evaluating training adaptation with heart-rate measures : A methodological comparison. Int J Sports Physiol Perform.2013;8(6), 688-691. https://doi.org/10.1123/ijspp.8.6.688

19. Buchheit M. Monitoring training status with HR measures: do all roads lead to Rome? Front $\quad$ Physiol. 2014;5, 1-19. https://doi.org/10.3389/fphys.2014.00073

20. Hopkins WG, Marshall SW, Batterham AM, Hanin J. Progressive statistics for studies in sports medicine and exercise science. Med Sci Sports Exerc. 2009;41(1): 3-13. https://doi.org/10.1249/MSS.0b013e31818cb278

21. Reynoso-Sánchez LF, Hoyos JR, García-Dávila M, Rosas AG, Jaenes JC, López-Walle J, Hernández-Cruz G. Cortisol y estrés-recuperación durante un periodo competitivo en jugadores de balonmano. Revista de Psicología del Deporte. 2017;26(suppl. 2): 125-131.

22. Buchheit M. Sensitivity of monthly heart rate and psychometric measures for monitoring physical performance in highly trained young handball players. Int J Sports Med.2015;36(5):351-356. https://doi.org/10.1055/s-0034-1385882

23. Thorpe R, Strudwick A, Buchheit M, Atkinson G, Drust B, Gregson W. Monitoring fatigue during the in-season competitive phase in elite soccer players. Int $\mathrm{J}$ Sports Physiol Perform.2015;10(8): 958-964. https://doi.org/10.1123/ijspp.2015-0004

24. Al Haddad H, Laursen PB, Collet D, Ahmaidi S, Buchheit M. Reliability of resting and postexercise heart rate measures. Int J Sports Med. 2011;32(8): 598-605. https://doi.org/10.1055/s-0031-1275356

25. Garrido A, de la Cruz B, Garrido MA, Medina M, Naranjo J. Variabilidad de la frecuencia cardiaca en un deportista juvenil durante una competición de máximo nivel. Rev Andal Med Deporte. 2009;2(2): 70-74.

26. Bonet J, Parrado E, Capdevila L. Efectos agudos del ejercicio físico sobre el estado de ánimo y la HRV. Rev.int.med.cienc.act.fís.deporte. 2017;17 (65): 85-100. https://doi.org/10.15366/rimcafd2017.65.006

27. Carvalho L, Milanez VF, Oliveira RS, Ramos SP, Leicht A, Nakamura FY. The influence of a weekend with passive rest on the psychological and autonomic recovery in professional male handball players. Kinesiology. 2015;47(1): 108114.

28. Kaikkonen P, Hynynen E, Mann T, Rusko H, Nummela A. Can HRV be used to evaluate training load in constant load exercises? Eur J Appl Physiol. 2010;108(3): 435-442. https://doi.org/10.1007/s00421-009-1240-1

29. Mougios V. Reference intervals for serum creatine kinase in athletes. Br J Sports Med. 2007;41(10): 674-678. https://doi.org/10.1136/bjsm.2006.034041

30. Chatzinikolaou A, Christoforidis C, Avloniti A, Draganidis D, Jamurtas A, Stampoulis T, Ermidis G, Sovatzidis A, Papassotiriou I, Kambas A, Fatouros I. A microcycle of inflammation following a team handball game. J Strength Cond Res. 2014;28(7): 1981-1994. https://doi.org/10.1519/JSC.0000000000000330 
31. de Moura NR, Cury-Boaventura MF, Santos VC, Levada-Pires AC, Bortolon J, Fiamoncini J, Pithon-Curi TC, Curi R, Hatanaka, E. Inflammatory response and neutrophil functions in players after a futsal match. J Strength Cond Res. 2012;26(9): 2507-2514. https://doi.org/10.1519/JSC.0b013e31823f29b5

32. Buchheit M, Voss SC, Nybo L, Mohr M, Racinais S. Physiological and performance adaptations to an in-season soccer camp in the heat: Associations with heart rate and heart rate variability. Scand J Med Sci Sports. 2011;21(6): 477-485. https://doi.org/10.1111/i.1600-0838.2011.01378.x

33. Souglis A, Bogdanis GC, Giannopoulou I, Papadopoulos C, Apostolidis N. Comparison of inflammatory response and muscle damage indices following a soccer, basketball, voleyball, \& handball game at an elite competitive level. Res Sports $\quad$ Med. 2015;23(1): https://doi.org/10.1080/15438627.2014.975814

34. Hartmann U, Mester J. Training and overtraining markers in selected sport events. Med Sci Sports Exerc. 2000;32(1): 209-215. https://doi.org/10.1097/00005768-200001000-00031

35. Olveira T, Gouveia MJ, Oliveira RF. Testosterone responsiveness to winning and lossing experience in female soccer players. Psychoneuroendocrinology.2009;34(7): 1056-1064. https://doi.org/10.1016/i.psyneuen.2009.02.006

36. Moreira A, Arati F, Lima-Arsati YB, Frachini E, de Araújo VC. Effect of a kickboxing match on salivary cortisol and immunoglobulin A. Percept Mot Skills. 2010;111(1):

158-166. https://doi.org/10.2466/05.06.16.25.PMS.111.4.158-166

37. Cunniffe B, Morgan KA, Baker JS, Cardinale M, Davies B. Home vs away competition: Effect on psychophysiological variables in elite rugby union. Int J Sports Physiol Perform. 2015;10(6): 687-694. https://doi.org/10.1123/ijspp.2014-0370

38. Chan S, Debono M. Replication of cortisol circadian rhythm: New advances in hydrocortisone and replacement therapy. Ther Adv Endocrinol Metab.2010;1(3): 129-138. https://doi.org/10.1177/2042018810380214

39. Maidana $\mathrm{P}$, Bruno OD, Mesch V. Medición de cortisol y sus fracciones: Una puesta al día. Medicina (B Aires).2013;73(6): 579-584.

40. Klaperski S, von Dawans B, Heinrichs M, Fuchs R. Effects of a 12-weeks endurance training program on the physiological response to psychosocial stress in men: A randomized controlled trial. J Behav Med. 2014;37(6): 11181133. https://doi.org/10.1007/s10865-014-9562-9

41. McLean BS, Coutts AJ, Kelly V, McGuigan MR, Cormack S. Neuromuscular, endocrine, and perceptual fatigue responses during different length betweenmatch microcycles in professional rugby league players. Int J Sports Physiol Perform. 2010;5(3): 367-383. https://doi.org/10.1123/ijspp.5.3.367

Número de citas totales / Total references: 41 (100\%) Número de citas propias de la revista / Journal's own references: $1(2,43 \%)$

Rev.int.med.cienc.act.fís.deporte - vol. 20 - número 77 - ISSN: 1577-0354 